\title{
Comparative analysis of radiation dose and low contrast detail detectability using routine paediatric chest radiography protocols
}

Objectives: To compare low contrast detail (LCD) detectability and radiation dose for routine paediatric chest X-ray (CXR) imaging protocols among various hospitals.

Methods: CDRAD 2.0 phantom and medical grade polymethyl methacrylate (PMMA) slabs were used to simulate the chest region of four different paediatric age groups. Radiographic acquisitions were undertaken on $17 \mathrm{X}$-ray machines located in eight hospitals using their existing CXR protocols. LCD detectability represented by image quality figure inverse ( $\left(\mathrm{QF}_{\text {inv }}\right)$ was measured physically using the CDRAD analyser software. Incident air kerma (IAK) measurements were obtained using a solid-state dosimeter.

Results: The range of $\mathrm{IQF}_{\text {inv }}$, between and within the hospitals, was 1.40-4.44 and 1.52-2.18, respectively for neonates; $0.96-4.73$ and $2.33-4.73$ for a 1 -year old; $0.87-1.81$ and $0.98-1.46$ for a 5-year old and 0.90-2.39 and 1.27-2.39 for a 10-year old.

The range of IAK, between and within the hospitals, was 8.56-52.62 $\mu \mathrm{Gy}$ and 21.79-52.62 $\mu \mathrm{Gy}$, respectively for neonates; 5.44-82.82 $\mu \mathrm{Gy}$ and 36.78-82.82 $\mu \mathrm{Gy}$ for a 1-year old; 10.97-59.22 $\mu \mathrm{Gy}$ and 11.75-52.94 $\mu$ Gy for a 5-year old and 13.97-100.77 $\mu$ Gy and 35.72-100.77 $\mu$ Gy for a 10-year old.

Conclusions: Results show considerable variation, between and within hospitals, in the LCD detectability and IAK. Further radiation dose optimisation for the four paediatric age groups, especially in hospitals /X-ray rooms with low LCD detectability and high IAK, are required.

\section{Keywords:}

Paediatric chest radiography, CDRAD phantom, low contrast detail detectability and radiation dose. 


\section{Introduction}

In radiography, the priority is to produce images with a sufficient level of quality to achieve diagnosis. However, the radiation dose to the patient should also be taken into consideration in order to avoid unnecessary radiation exposure [1]. The importance of this is even more apparent for paediatric radiology since children are up to a factor of 10 more radiosensitive than adults [2,3]. By keeping the radiation exposure as low as reasonably practicable (ALARP), both professionals and legislators strive to minimise this risk while maintaining image quality (IQ). The balance between radiation dose and IQ is often referred to as optimisation and is one of the fundamental principles of radiographic practice [4]. Optimisation is not easy to achieve because of differences in imaging systems performance, patient size variations and differences in clinical imaging protocols that are in routine use. Paediatric dose optimisation is even more challenging than that for adults due to the wide variation in paediatric sizes which makes the determination of optimum exposure parameters more difficult [4,5]. The above factors could lead to IQ and radiation dose differences, between and within hospitals, for the same clinical investigations. IQ differences may affect the diagnostic accuracy. Furthermore, differences in radiation dose affect the risk to the patient.

Among the different X-ray examinations, chest radiography (CXR) is the most common examination in children as a result of common respiratory conditions e.g. pneumonia and is also invaluable for resolving a broad range of clinical problems [6-8]. However, the optimisation of IQ and radiation dose for CXR is considered to be difficult because of the wide range of tissue types and medical indications within the chest region. These tissue types vary between high (e.g. mediastinum) and low (e.g. lung) X-ray attenuation characteristics [9,10]. Often there are a number of clinical protocols that are available and suitable for undertaking CXRs [10]. Consequently, questions have arisen as to what extent do standard clinical protocols for undertaking paediatric X-ray examinations vary between imaging systems and departments and what is the resultant impact on IQ and radiation dose? In the United Kingdom (UK), there is a lack of data about the likely differences, between and within hospitals, in terms of radiation dose and IQ for paediatric CXRs. The aim of this study is to compare low contrast detail (LCD) detectability and radiation dose for paediatric CXR protocols across a selection of hospitals using the CDRAD 2.0 phantom. 


\section{Method}

A CDRAD 2.0 phantom (Artinis Medical System, The Netherlands, Fig. 1) accompanied with medical grade polymethyl methacrylate (PMMA) slabs were used to investigate the variations in LCD detectability for seventeen diagnostic X-ray machines located in eight UK National Health Service (NHS) hospitals within the North-west of England. An evaluation of radiation dose was also conducted using the same phantom and a solid-state radiation detector. All X-ray machines used in this study passed quality control tests based on the Institute of Physics and Engineering in Medicine (IPEM) report 91[11].

\subsection{Phantom placement and image acquisition}

In order to simulate the chest size of four different paediatric age groups, the CDRAD 2.0 phantom was placed between different thicknesses of PMMA slabs. Using a conversion factor, $1 \mathrm{~cm}$ of PMMA equated to $1.5 \mathrm{~cm}$ of the chest region, chest sizes of four age groups were simulated $[12,13]$. The groups were defined as neonate, 1,5 and 10-years old with respective chest thicknesses of 9.8, 13.0, 15.0 and $16.8 \mathrm{~cm}$ [14]. The phantom was positioned at the centre of primary radiation field and the X-ray beam was collimated to the edges of the phantom. Using the standard protocols, for each X-ray unit / hospital, corresponding to the age groups, shown in Table 1. three repeated images were acquired for each acquisition, as recommended by the CDRAD 2.0 phantom manufacturer [15]. For exposures produced with the automatic exposure control (AEC) the post exposure mAs values were also noted in Table 1. Hospitals $(\mathrm{H})$ and X-ray machines $(\mathrm{X})$ were separately coded (i.e. hospital 1 and machine 1 is shown as H1X1). Post-processing was determined locally based on the routine chest imaging protocol for each X-ray machine / hospital. The reason for this choice was to increase the ecological validity of the study by including all the factors that can lead to the variation in IQ between and within hospitals.

\subsection{LCD detectability evaluation}

Using the CDRAD phantom analyser software version 2.1 the LCD detectability was measured automatically and represented as image quality figure inverse (IQFinv) [15]. Other studies [e.g. 16,17] have demonstrated that there is good correlation between visual (human observers) and physical (using analyser software) scoring methods when evaluating LCD detectability, represented by $\mathrm{IQF}_{\text {inv }}$, for CDRAD 2.0 phantom images. 
The $\mathrm{IQF}_{\text {inv }}$ was calculated from the summation of the product of the lowest hole diameter (threshold diameter) in each of the fifteen columns where the hole is correctly detected and their intrinsic depths (contrast) using equation (1).

$$
I Q F_{i n v}=\sum_{i=1}^{15} \frac{1}{C_{i} D_{(i, t h)}}
$$

$\mathrm{D}_{(\mathrm{i}, \text { th) }}$ represents the lowest diameter in column (i) that has a visible hole correctly detected; $\mathrm{C}_{\mathrm{i}}$ represents the contrast of the threshold visible hole in the column (i).

The software parameters needed for analysis (i.e. alpha level of significance, a priori difference of the mean (APD) and source image distance (SID)) were predetermined based on literature or protocol. Alpha was selected to be equal to $1 \times 10^{-8}$. This value is equal to the default value of the CDRAD version 2.1 analyser software and is proposed by the manufacturer [15]. The reason behind selecting this value is attributed to it having the best correlation with perceptual IQF inv $_{\text {[18]. }}$ APD should be included in the calculation during the automated scoring method and it should be set relative to the image bit depth [15]. Within this study, APD was set to 0 because the images had different bit depths, since they were acquired from different X-ray machines, to ensure a valid comparison between images $[15,19]$. SID values were set depending on the local imaging protocol.

\subsection{Dosimetry}

Using a solid-state dosimeter (RaySafe X2, Unfors Ray Safe AB, Billdal, Sweden) incident air kerma (IAK) was measured for all manual exposures. The dosimeter was placed at the surface of the phantom in line with the central beam. All exposures were repeated threefold and the average value was calculated. For examinations which used the AEC a simple mathematical method was used to estimate IAK. This technique was based on the estimation of IAK values from a series of post-exposure mAs values recorded as part of a calibration procedure. For a series of manual exposures IAK values were recorded against their respective mAs value using the solid-state dosimeter. From the graphical representation of IAK versus mAs, a best fit line and resultant regression equation were generated to provide a method for estimating the IAK from post-exposure mAs values obtained from the phantom when the AEC was in use. 


\subsection{Data analysis}

Data were analysed using SPSS version 22.0 (IBM Inc, Armonk, New York, US) and were first examined to investigate the normality using the Shapiro-Wilk test. Tests confirmed that the IQFinv for the neonate, 1-year and 5-years age group and the IAK for the neonate and 1-year group had an normal distribution ( $\mathrm{p}>0.05$ ) ; while the $\mathrm{IQF}_{\text {inv }}$ for the 10 -years old group and the IAK for the 5-years and 10-years old groups had a non-normal distribution $(\mathrm{p}<0.05)$. Pearson's correlation $(\mathrm{r})$ coefficients were used for investigating the correlation between the scores of IQF $\mathrm{F}_{\text {inv }}$ and IAK for the parametric data; while Spearman's correlation was used for the nonparametric data. The correlation ( $r$ ) was considered small for $r=0.1$ to 0.29 , medium for $r=0.30$ to 0.49 , and large for $\mathrm{r}=0.50$ to $1.0[20,21]$.

\section{Results}

Overall, the results demonstrate a large variation in $\mathrm{IQF}_{\text {inv }}$ and IAK between and within hospitals (Figs. 2-5). However, the extent of this variation is different between the age groups. Table 2 provides a summary of the key results represented by the minimum and maximum values of the IAK and $\mathrm{IQF}_{\text {inv }}$ and their percentage difference, between and within hospitals and the mean/median between the hospitals is also presented. Table 1 highlights that the hospitals did not make use of an anti-scatter grid, and the inclusion of the AEC increased when imaging 10 year olds. In addition, the variations in exposure parameters for the neonate age group were lower than that of the other age groups; the percentage difference of the $\mathrm{kVp}$ and $\mathrm{mAs}$ values among $\mathrm{X}$-ray units for the neonate, 1, 5 and 10-years old were 12.5\%, 19.5\%, 62.2\%, 59.4\% and $104.1 \%, 85.7 \%$, $111.1 \%, 137.4 \%$, respectively. Furthermore, most of the hospitals did not use additional filtration. Correlations between IQFinv and IAK for neonates, 1-year, 5-years and 10-years age groups were $(\mathrm{r}=0.54 ; \mathrm{p}=0.02),(\mathrm{r}=0.20 ; \mathrm{p}=0.44),(\mathrm{r}=0.24 ; \mathrm{p}=0.35)$ and $(\mathrm{r}=0.10 ; \mathrm{p}=0.71)$, respectively. 


\section{Discussion}

The aim of this study was to investigate whether differences in IQ and radiation dose occur between and within various hospitals. This study showed a large variation in both IQFinv and IAK and thus a lack of standardisation for some of the acquisition parameters. As a consequence, a wide degree of IQ and radiation dose variation occurs, both between and within hospitals.

Within the neonatal age group, 4 out of 17 machines produced $\mathrm{IQF}_{\text {inv }}$ values above the $3^{\text {rd }}$ quartile (3.18). Of the 17 machines, 4 (H5X1, H6X1, H6X2, H8X1) had an IQFinv below the $1^{\text {st }}$ quartile (1.86). X-ray machine H2X4 shows a notably high $\mathrm{IQF}_{\text {inv }}$ (4.44), and a high IAK (52.62 $\left.\mu \mathrm{Gy}\right)$. This is most likely due to the high mAs used (2 mAs) since increasing mAs lead to increase IAK and $\mathrm{IQF}_{\text {inv }}$ and vice versa, especially when compared to other machines and is in concordance with previous studies [22-25]. In contrast, the low IQF inv for H6X1 and H6X2 is likely to be caused by the relatively high SID which, due to the inverse square law, decreases the IAK (8.56 and 8.57 $\mu \mathrm{Gy}$, respectively). This lower IAK results in a decrease in signal to noise ratio (SNR) which negatively influences the IQFinv. In addition to the high SID and both the mAs and $\mathrm{kVp}$ values used are relatively low compared to other machines, which reduces the $\mathrm{IQF}_{\text {inv }}$ even further. In addition to the variation in the acquisition parameters among the X-ray units, the lack of additional filtration in most protocols is also worthy of note as the use of additional filtration reduces the IAK while having a minimal influence on the $\mathrm{IQF}_{\text {inv }}$ as shown by the results from $\mathrm{H} 3 \mathrm{X} 1-5$. This is especially important since the International Commission on Radiological Protection (ICRP) has recommended the use of additional filtration in regard to paediatric imaging in ICRP publication 121. Several studies have also recommended utilising additional filtration for both paediatric and adult acquisitions and have concluded that employing additional filtration can reduce radiation dose but without reduction IQ [19,26,27]. Reasons for not adopting additional filtration within this study may relate to all study institutions being general hospitals and not specifically focused on the imaging of paediatrics. It must be acknowledged that identical IAK values on two different X-ray units, with different tube potentials and different levels of filtration, could potentially generate differences in effective dose. Results from our experimental work should take into account additional filtration and $\mathrm{kVp}$ settings when interpreting IAK values.

The relation between radiation dose and $\mathrm{IQF}_{\text {inv }}$ was also demonstrated within the 1-year age group. As with the neonate group, H2X4 shows the highest IQFinv (4.73), highest IAK (82.82 $\mu$ Gy), highest mAs $(2.5 \mathrm{mAs})$ and shortest SID $(115 \mathrm{~cm})$. The second highest IAK $(72.47 \mu \mathrm{Gy})$ also 
follows this trend with a high mAs $(1.8 \mathrm{mAs})$ and low SID $(120 \mathrm{~cm})$. Incidentally, the three IQFinv scores below the $1^{\text {st }}$ quartile (1.66) show similarities between each other. The two lowest IQFinv (H4X1; 1.40 and H4X2; 0.96) had a relatively high SID (180 cm) and low mAs (1 mAs). The difference in $\mathrm{IQF}_{\text {inv }}$ between these two machines is likely the result of different detector types used (digital radiography [DR] and computed radiography [CR], respectively) and filtration (' $1 \mathrm{~mm} \mathrm{Al}$ $+0.1 \mathrm{~mm} \mathrm{Cu}$ ' and none, respectively). Lastly, H8X1 again makes use of a CR detector in combination with a low mAs (1.25 mAs). Several phantom studies, based on the CDRAD 2.0, have shown that the LCD detectability performance of DR systems are better than that of CR systems, for the same radiation dose level [19,26].

With regards to the 5-year age group, four of the sixteen X-ray machines were found to pass the

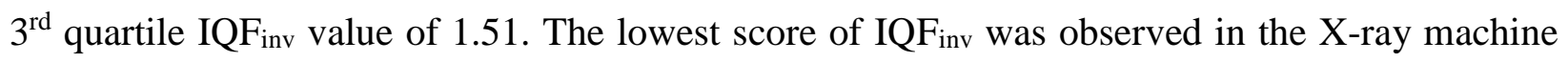
H8X1 (0.87). This score is most likely caused by a relative low mAs (1.25 mAs) in combination with a high SID $(200 \mathrm{~cm})$ and a CR detector. In this age group (and in the 10-year old group) there is also an increase in AEC use, this may reflect recommendations by the Commission of the European Communities (CEC) [28] who do not recommended AECs for the infants and young children, even though the CEC guidelines were designed for film screen systems and not for digital systems. It should be noted that implementing the use of the AEC could lead to an increase in dose as shown by H6X1 and H6X2, which up until this age group had a relatively low dose. This is in concordance with previous studies which conclude that AEC is associated with higher radiation dose for paediatrics when compared with the manual control $[29,30]$. The likely reason for delivering higher radiation dose with the AEC for paediatric compared with that of the manual control may be because most AEC systems are not designed specifically for paediatric patients. These systems have comparatively large and fixed ionisation chambers and their size, shape, and location do not reflect the differences in body size in paediatric patients [30].

This effect is not shown in $\mathrm{H} 2 \mathrm{X} 3$, which also implements the AEC at with this age group. This is most likely caused by the large increase in tube voltage. The use of additional filtration is further demonstrated by $\mathrm{H} 3 \mathrm{X} 2$ and $\mathrm{H} 3 \mathrm{X} 3$ which demonstrate a relatively good $\mathrm{IQF}_{\text {inv }}$ with the lowest IAK measured, 11.00 and $10.97 \mu \mathrm{Gy}$, respectively.

Finally, for the 10-year age group, from the sixteen X-ray machines, four scores were observed to be higher than the $3^{\text {rd }}$ quartile (1.73). An outlier value of $\mathrm{IQF}_{\text {inv }}$ was observed for both $\mathrm{H} 2 \mathrm{X} 1$ and $\mathrm{H} 2 \mathrm{X} 2$ with respective values of 2.39 and 2.24 that could not be explained by the H2X1 protocol. 
However, H2X2 has a mAs value double that of most other systems ( 5 mAs) which resulted in an extremely high IAK (100.77 $\mu \mathrm{Gy})$. The X-ray machine H8X1 was observed to have the lowest score of $\operatorname{IQF}_{\text {inv }}(0.90)$, this might be attributed to the high SID value $(200 \mathrm{~cm})$ in combination with the detector type being CR.

In addition, the variability in $\mathrm{IQF}_{\text {inv }}$ values among $\mathrm{X}$-ray machines could also be attributed to the type of the image processing used, since different types of image processing were utilised as part of the routine imaging protocol for each X-ray machine/hospital.

A good correlation was observed between the IAK and $\mathrm{IQF}_{\text {inv }}$ for only the neonatal age group. Only a weak, non-statistically significant, correlations were observed between IAK and IQF $F_{\text {inv }}$ for the 1-year, 5-years and 10-years age groups. These results could relate to the different technical characteristics of the machines (detector system characteristics), exposure parameters and technique used for the same examination. The results provide evidence that there is opportunity for further optimisation regarding radiation dose reduction without reducing LCD detectability. The variability of the acquisition parameters for the neonate age group among the X-ray units was less than that for the other age groups. This low variability can be the reason behind obtaining a good correlation $(\mathrm{r}=0.54)$ between $\mathrm{IAK}$ and $\mathrm{IQF}_{\text {inv }}$ in this age group compared with the other age groups which had a weak correlation. This might be related to the lack of variations in the size of a neonate when compared with children in other age groups, this provides an indication to the possibility of standardisation of routine protocols in neonatal imaging.

There is a tendency for $\mathrm{IQF}_{\text {inv }}$ to decline as age / size increases and this can be seen from the mean values of $\mathrm{IQF}_{\text {inv }}$ across the different age groups (Table 2). Such changes in $\mathrm{IQF}_{\text {inv }}$ can be explained by the increasing PMMA thickness (simulating increased patient size) when increasing the phantom age groups. Increasing the PMMA thickness leads to an increase in scattered radiation which decreases SNR [31] and then $\mathrm{IQF}_{\text {inv }}$ values. Therefore, it is not valid to compare the numerical values of the IQFinv between the different age groups because their PMMA thickness is different and a valid comparison needs be achieved within each group independently.

Our recommendation supporting utilising an additional filtration in paediatric CXR examination since it has a large influence on the patient dose reduction while maintaining LCD detectability and this also has been recommended by the CEC for paediatrics. 
In our study the IQ is represented by the LCD detectability using the CDRAD 2.0 phantom which is a widely used method for assessing and comparing imaging systems and for undertaking optimisation studies. [6,32-35]. Clinically, improvement of LCD detectability could be beneficial, particularly in chest radiography, for increasing the detectability of lesions and lung disease. The physical approach of assessment LCD detectability represented by IQFinv using CDRAD 2.0 phantom was reported to have an excellent correlation with the visual IQ evaluations, as noted by De Crop et al. [16]. More recently, a study undertaken by our research group confirmed the validity of physical evaluation for LCD detectability using CDRAD 2.0 phantom for evaluating visual IQ and lesion visibility along with its validity in CXR optimisation studies [36]. Within this work it was concluded that there is a good correlation between the LCD detectability and the visibility of the lesions and the general visual IQ within CXR. Two spherical lesions that simulate nodules of different sizes that were placed in different locations that had different visibilities were used within the study: 1) $10 \mathrm{~mm}+100 \mathrm{HU}$ (Hounsfield unit) lesion located in left upper lobe. 2) The $12 \mathrm{~mm}$ $+100 \mathrm{HU}$ lesion was located in the right middle lobe. A good positive correlation was observed between $\mathrm{IQF}_{\text {inv }}$ and lesion visibility for the two simulated lesions; the first lesion showed $\mathrm{r}=0.79$ $(\mathrm{p}<0.001)$ and the second lesion showed $\mathrm{r}=0.68(\mathrm{p}<0.001)$. A strong positive correlation $(\mathrm{r}=0.91$; $\mathrm{p}<0.001$ ) between the IQF $\mathrm{Fin}_{\text {v }}$ and visual IQ evaluations from the Lungman phantom was observed. Consequently, utilising the LCD detectability represented by $\mathrm{IQF}_{\text {inv }}$, with CDRAD 2.0 phantom, as a metric for IQ assessment in our study was consequently justified.

Therefore, the clinical effects of the observed differences in LCD detectability in our current study, especially for the X-ray units with the lowest values of LCD detectability, should be taken into account in future work to help increasing the pathology detection performance. These differences in LCD detectability could influence the detection of subtle pathology in clinical practice. More research is necessary to investigate how such differences in LCD detectability can influence pathology detection. If the variations in LCD detectability reported in our study do not have a negative impact on the diagnostic accuracy of pathology, then further reduction of the radiation dose can be achieved. Our findings and subsequent recommendations regarding how optimisating routine imaging could occur should be confirmed by anthropomorphic phantom studies or clinical IQ studies to consider the limitations of the phantom (e.g. having a uniform background).

A relatively small number of hospitals and X-ray machines were involved in our study; however, it is sensible to speculate that our findings are applicable for paediatric CXR examinations in 
general. This is because paediatric CXR examinations are difficult examinations in which to achieve LCD detectability and dose optimisation, as such variations in LCD detectability and radiation dose are likely to exist in other imaging centres/hospitals which conduct paediatric CXR examinations. By considering that differences in routine clinical protocols for paediatric CXR examinations exist and in the absence of standardised imaging protocols, we suggest that paediatric CXR examinations should be evaluated in all hospitals to ensure that the IQ is optimal and sufficient for diagnosis, while radiation dose as low as possible to minimise risk to patients.

A limitation of our study is the type of X-ray machines utilised for imaging the neonates since these were mainly stationary X-ray machines while neonatal CXR examinations are likely to be conducted using mobile X-ray machines. Future work should be conducted using a large number of mobile X-ray machines. Our study should also be extended to include investigating the influence of variations in LCD detectability, between and within hospitals, on pathology identification / diagnostic performance in clinical practice.

\section{Conclusion}

Our study clearly demonstrates that standard clinical protocols for paediatric CXR examinations are not sufficiently optimised. As a result, a wide difference in LCD detectability and IAK between the participating hospitals was observed. Our findings indicate that the paediatric CXR acquisition factors should be investigated in all the hospitals /imaging centres to optimise routine imaging protocols and ensuring that the IQ is acceptable for diagnosis and radiation dose as low as possible.

\section{Declarations of interest: none.}




\section{References}

[1] H.P. Busch, K. Faulkner, Image quality and dose management in digital radiography: a new paradigm for optimisation, Radiat. Prot. Dosimetry. 117 (2005) 143-147. doi:10.1093/rpd/nci728.

[2] M.T. Raissaki, Pediatric radiation protection, Eur. Radiol. Suppl. 14 (2004) 74-83. doi:10.1007/s10406-004-0011-7.

[3] J.D. Mathews, A. V. Forsythe, Z. Brady, M.W. Butler, S.K. Goergen, G.B. Byrnes, G.G. Giles, A.B. Wallace, P.R. Anderson, T.A. Guiver, P. McGale, T.M. Cain, J.G. Dowty, A.C. Bickerstaffe, S.C. Darby, Cancer risk in 680000 people exposed to computed tomography scans in childhood or adolescence: data linkage study of 11 million Australians, BMJ. 346 (2013) f2360-f2360. doi:10.1136/bmj.f2360.

[4] C.E. Willis, Optimizing digital radiography of children, Eur. J. Radiol. 72 (2009) 266-273. doi:10.1016/j.ejrad.2009.03.003.

[5] a. Almen, M. Lööf, S. Mattsson, Examination Technique, Image Quality, and Patient Dose in Paediatric Radiology, Acta Radiol. 37 (1996) 337-342. doi:10.1177/02841851960371P171.

[6] K. Bacher, P. Smeets, K. Bonnarens, A. De Hauwere, K. Verstraete, H. Thierens, Dose Reduction in Patients Undergoing Chest Imaging: Digital Amorphous Silicon Flat-Panel Detector Radiography Versus Conventional Film-Screen Radiography and Phosphor-Based Computed Radiography, Am. J. Roentgenol. $181 \quad$ (2003) 923-929. doi:10.2214/ajr.181.4.1810923.

[7] W.J.H. Veldkamp, L.J.M. Kroft, J. Geleijns, Dose and perceived image quality in chest radiography, Eur. J. Radiol. 72 (2009) 209-217. doi:10.1016/j.ejrad.2009.05.039.

[8] S. Raoof, D. Feigin, A. Sung, S. Raoof, L. Irugulpati, E.C. Rosenow, Interpretation of Plain Chest Roentgenogram, Chest. 141 (2012) 545-558. doi:10.1378/chest.10-1302.

[9] P. Doyle, C.J. Martin, D. Gentle, Application of contrast-to-noise ratio in optimizing beam quality for digital chest radiography: comparison of experimental measurements and theoretical simulations, Phys. Med. Biol. 51 (2006) 2953-2970. doi:10.1088/00319155/51/11/018.

[10] G. Singh, C.J. Martin, A. McCurrach, G. Phanco, Optimisation of performance for computed radiography in the West of Scotland, Radiat. Prot. Dosimetry. 154 (2013) 293300. doi:10.1093/rpd/ncs238.

[11] Institute of Physics and Engineering in Medicine, IPEM report 91: recommended standards for the routine performance testing of diagnostic x-ray imaging systems, IPEM, York, 2005.

[12] H. Bosmans, J. Nens, L. Delzenne, N. Marshall, H. Pauwels, W. De Wever, R. Oyen, Exploration of exposure conditions with a novel wireless detector for bedside digital radiography, in: N.J. Pelc, R.M. Nishikawa, B.R. Whiting (Eds.), Phys. Med. Biol., 2012: p. 83135 K. doi: $10.1117 / 12.911588$.

[13] J. Rassow, A.A. Schmaltz, F. Hentrich, C. Streffer, Effective doses to patients from 
paediatric cardiac catheterization., Br. J. Radiol. 73 (2000) 172-183. doi:10.1259/bjr.73.866.10884731.

[14] M. Tapiovaara, T. , Siiskonen, A Monte Carlo program for calculating patient doses in medical x-ray examinations, 2nd ed., Radiation and Nuclear Safety Authority, Helsinki,Finland, 2008.

[15] R. van der Burght, M. Floor, M. Thijssen, R. Bijkerk, Manual CDRAD 2.0 phantom and analyser software version 2.1, Artinis Medical Systems, Einsteinweg 17 , The Netherlands, 2014.

[16] A. De Crop, K. Bacher, T. Van Hoof, P. V Smeets, B.S. Smet, M. Vergauwen, U. Kiendys, P. Duyck, K. Verstraete, K. D'Herde, H. Thierens, Correlation of Contrast-Detail Analysis and Clinical Image Quality Assessment in Chest Radiography with a Human Cadaver Study, Radiology. 262 (2012) 298-304. doi:10.1148/radiol.11110447.

[17] J.M. Fernandez, J.M. Ordiales, E. Guibelalde, C. Prieto, E. Vano, Physical image quality comparison of four types of digital detector for chest radiology, Radiat. Prot. Dosimetry. 129 (2008) 140-143. doi:10.1093/rpd/ncn026.

[18] a Pascoal, C.P. Lawinski, I. Honey, P. Blake, Evaluation of a software package for automated quality assessment of contrast detail images - comparison with subjective visual assessment, Phys. Med. Biol. 50 (2005) 5743-5757. doi:10.1088/0031-9155/50/23/023.

[19] P. Brosi, A. Stuessi, F.R. Verdun, P. Vock, R. Wolf, Copper filtration in pediatric digital Xray imaging: its impact on image quality and dose, Radiol. Phys. Technol. 4 (2011) 148155. doi:10.1007/s12194-011-0115-4.

[20] J. Cohen, Statistical power analysis for the behavioral sciences.Hilsdale." NJ: Lawrence Earlbaum Associates 2, 1988.

[21] A. Filed, Discovering statistics using SPSS statistics, Third edit, Sage publications Ltd, Londan, 2009.

[22] A. De Hauwere, K. Bacher, P. Smeets, K. Verstraete, H. Thierens, Analysis of image quality in digital chest imaging, Radiat. Prot. Dosimetry. 117 (2005) 174-177. doi:10.1093/rpd/nci748.

[23] H. Alsleem, P. U, K.S. Mong, R. Davidson, Effects of radiographic techniques on the lowcontrast detail detectability performance of digital radiography systems, Radiol. Technol. 85 (2014) 614-22.

[24] Z. Sun, C. Lin, Y. Tyan, K.H. Ng, Optimization of chest radiographic imaging parameters: A comparison of image quality and entrance skin dose for digital chest radiography systems, Clin. Imaging. 36 (2012) 279-286. doi:10.1016/j.clinimag.2011.09.006.

[25] E. Seeram, R. Davidson, S. Bushong, H. Swan, Optimizing the Exposure Indicator as a Dose Management Strategy in Computed Radiography., Radiol. Technol. 87 (2016) 380391.

[26] E.U. Ekpo, A.C. Hoban, M.F. McEntee, Optimisation of direct digital chest radiography using Cu filtration, Radiography. 20 (2014) 346-350. doi:10.1016/j.radi.2014.07.001. 
[27] O.W. Hamer, M. Völk, N. Zorger, I. Borisch, R. Büttner, S. Feuerbach, M. Strotzer, Contrast-detail phantom study for $x$-ray spectrum optimization regarding chest radiography using a cesium iodide-amorphous silicon flat-panel detector., Invest. Radiol. 39 (2004) 610618. doi:00004424-200410000-00004 [pii].

[28] Commission of the European Communities (CEC), European Guidelines on quality criteria for diagnostic radiographic images in paediatrics:(UR 16261EN)., Luxembourg: CEC, 1996. ftp://ftp.cordis.lu/pub/fp5-euratom/docs/eur16261.pdf.

[29] A. Almén, M. Lööf, S. Mattsson, Examination Technique, Image Quality, and Patient Dose in Paediatric Radiology, Acta Radiol. 37 (1996) 337-342. doi: $10.3109 / 02841859609177662$.

[30] K. Hintenlang, J. Williams, D. Hintenlang, A survey of radiation dose associated with pediatric plain-film chest X-ray examinations, Pediatr. Radiol. 32 (2002) 771-777. doi:10.1007/s00247-002-0734-3.

[31] M.B. Williams, E.A. Krupinski, K.J. Strauss, W.K. Breeden, M.S. Rzeszotarski, K. Applegate, M. Wyatt, S. Bjork, J.A. Seibert, Digital Radiography Image Quality: Image Acquisition, J. Am. Coll. Radiol. 4 (2007) 371-388. doi:10.1016/j.jacr.2007.02.002.

[32] H. Geijer, K.-W. Beckman, T. Andersson, J. Persliden, Image quality vs radiation dose for a flat-panel amorphous silicon detector: a phantom study, Eur. Radiol. 11 (2001) 17041709. doi:10.1007/s003300100851.

[33] X.J. Rong, C.C. Shaw, X. Liu, M.R. Lemacks, S.K. Thompson, Comparison of an amorphous silicon/cesium iodide flat-panel digital chest radiography system with screen/film and computed radiography systems - A contrast-detail phantom study, Med. Phys. 28 (2001) 2328-2335. doi:10.1118/1.1408620.

[34] W.J.H. Veldkamp, L.J.M. Kroft, M. V. Boot, B.J.A. Mertens, J. Geleijns, Contrast-detail evaluation and dose assessment of eight digital chest radiography systems in clinical practice, Eur. Radiol. 16 (2006) 333-341. doi:10.1007/s00330-005-2887-6.

[35] K. Alzimami, S. Sassi, M. Alkhorayef, A.J. Britten, N.M. Spyrou, Optimisation of computed radiography systems for chest imaging, Nucl. Instruments Methods Phys. Res. Sect. A Accel. Spectrometers, Detect. Assoc. Equip. 600 (2009) 513-518. doi:10.1016/j.nima.2008.12.023.

[36] S. Al-Murshedi, P. Hogg, A. England, An investigation into the validity of utilising the CDRAD 2.0 phantom for optimisation studies in digital radiography, Br. J. Radiol. 91 (2018) 20180317. doi:10.1259/bjr.20180317. 
Figures and tables:

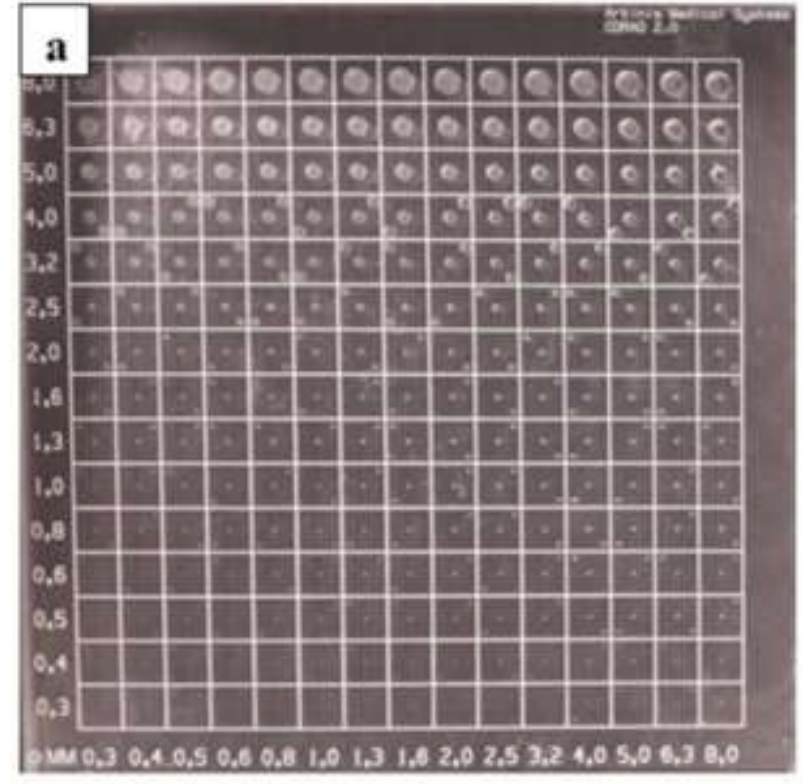

Fig. 1a. CDRAD 2.0 phantom.

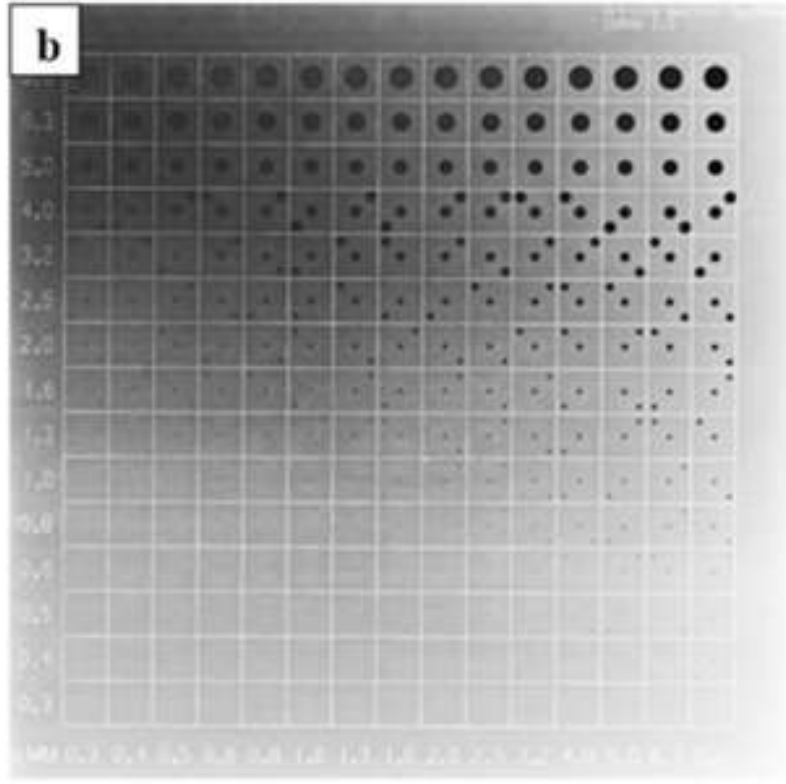

Fig. 1b. Resultant CDRAD 2.0 X-ray image.

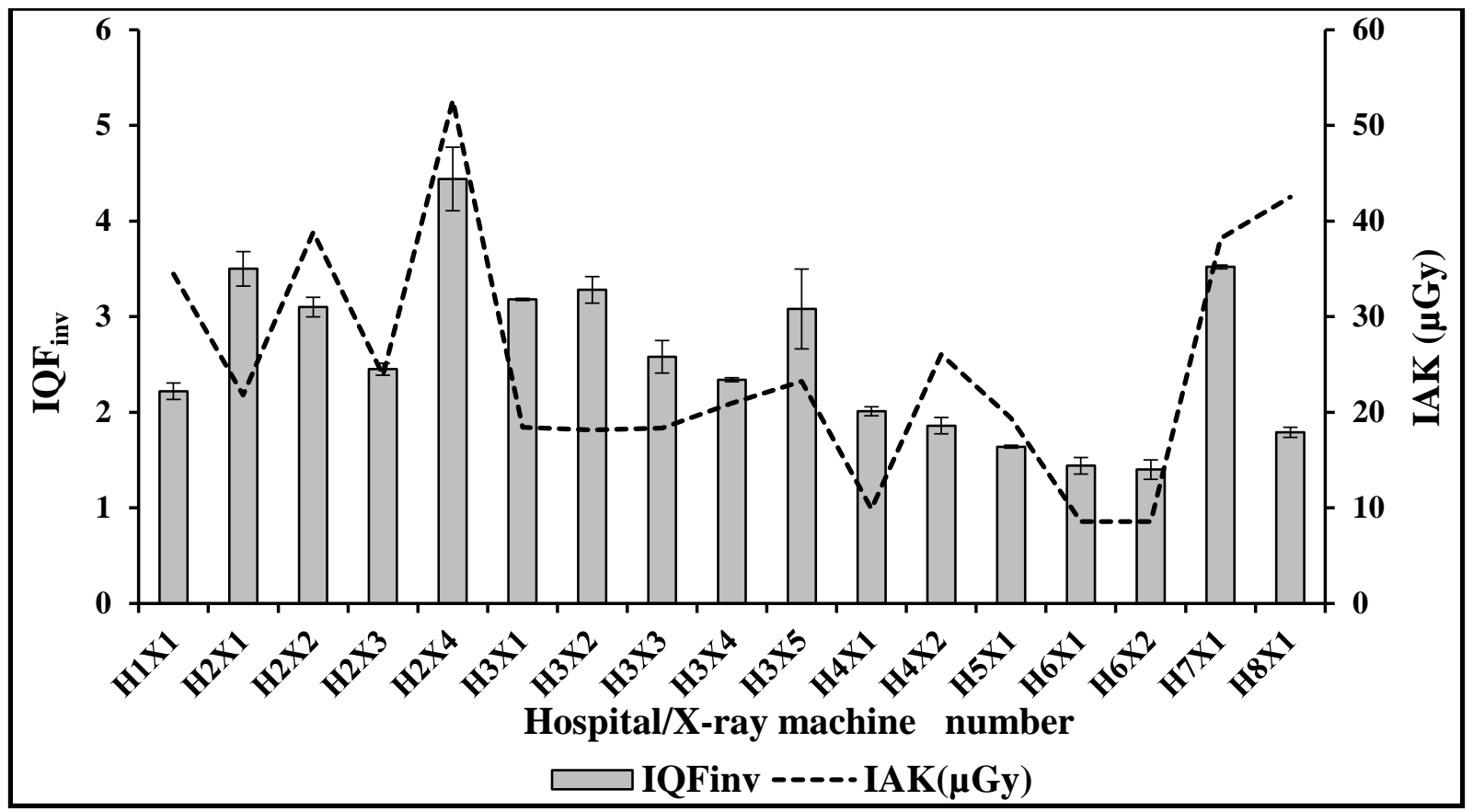


Fig. 2. A comparison of $I Q F_{i n v}$ against the $I A K$ for the neonate age group, between/within hospitals. $\mathrm{H}=$ hospital; $\mathrm{X}=\mathrm{X}$-ray unit. The bars represent the $\mathrm{IQF}_{\text {inv }}$ values with the accompanying error bars representing the standard deviation (SD) of three replicated images. The dotted line represents the IAK values.

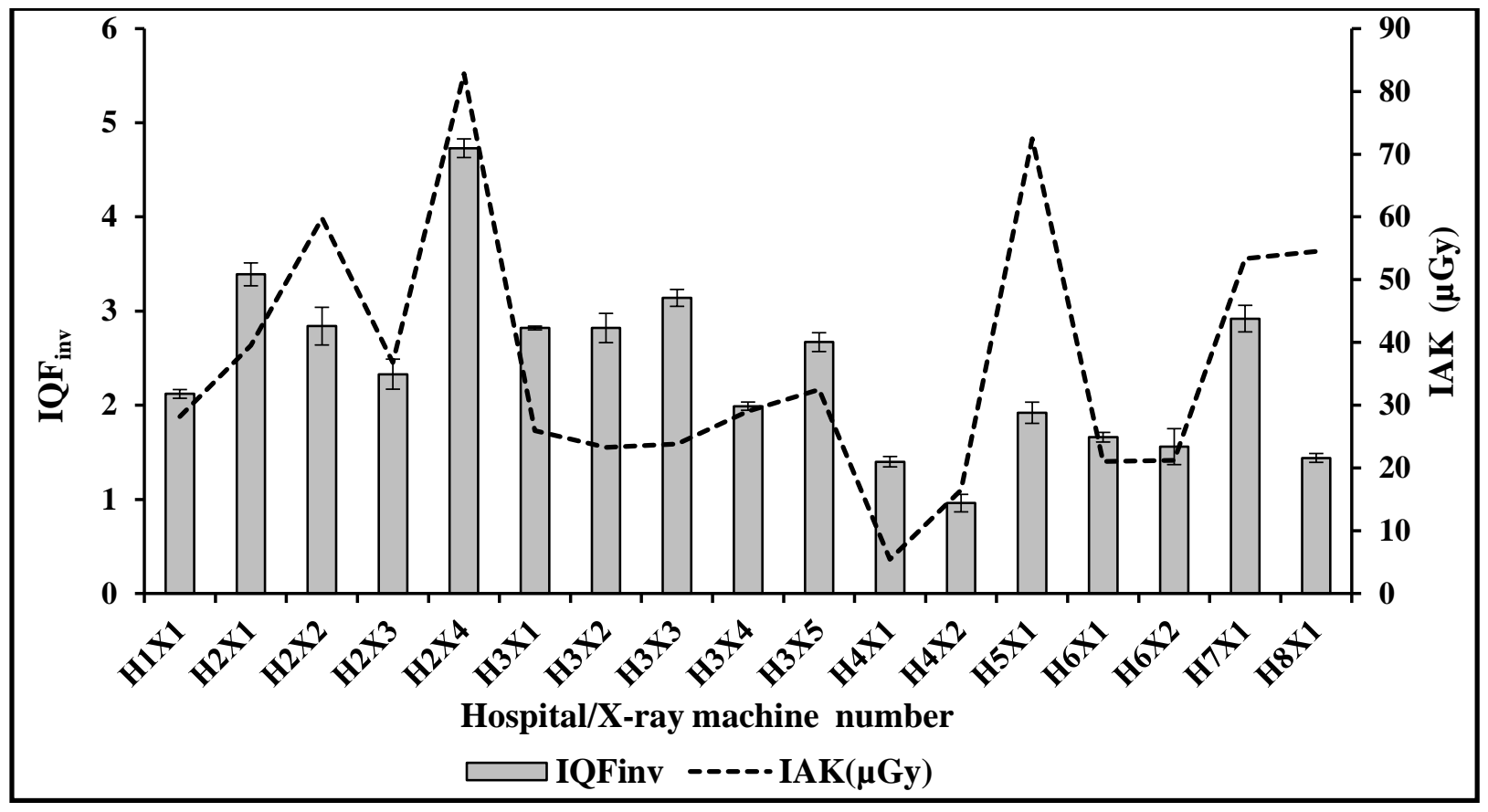

Fig. 3. A comparison of IQF $F_{\text {inv }}$ against the IAK for the one year age group, between /within hospitals. $\mathrm{H}=$ hospital; $\mathrm{X}=\mathrm{X}$-ray unit. The bars represent the $\mathrm{IQF}_{\text {inv }}$ values with the accompanying error bars representing the SD of three replicated images. The dotted line represents the IAK values. 


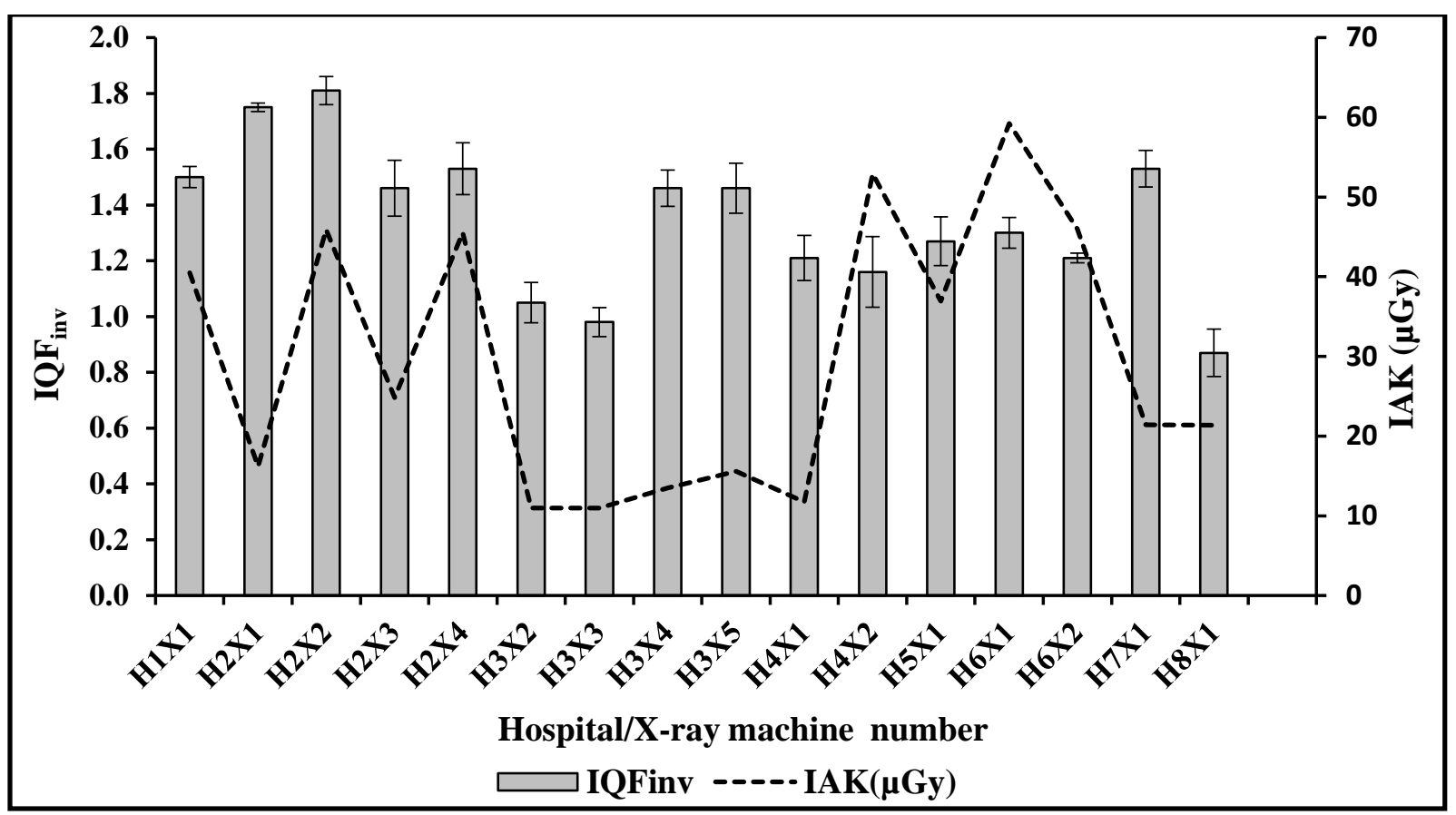

Fig. 4. A comparison of $I_{Q} F_{\text {inv }}$ against the IAK for the five years age group, between /within hospitals. $\mathrm{H}=$ hospital; $\mathrm{X}=\mathrm{X}$-ray unit. The bars represent the $\mathrm{IQF}_{\text {inv }}$ values with the accompanying error bars representing the SD of three replicated images. The dotted line represents the IAK values.

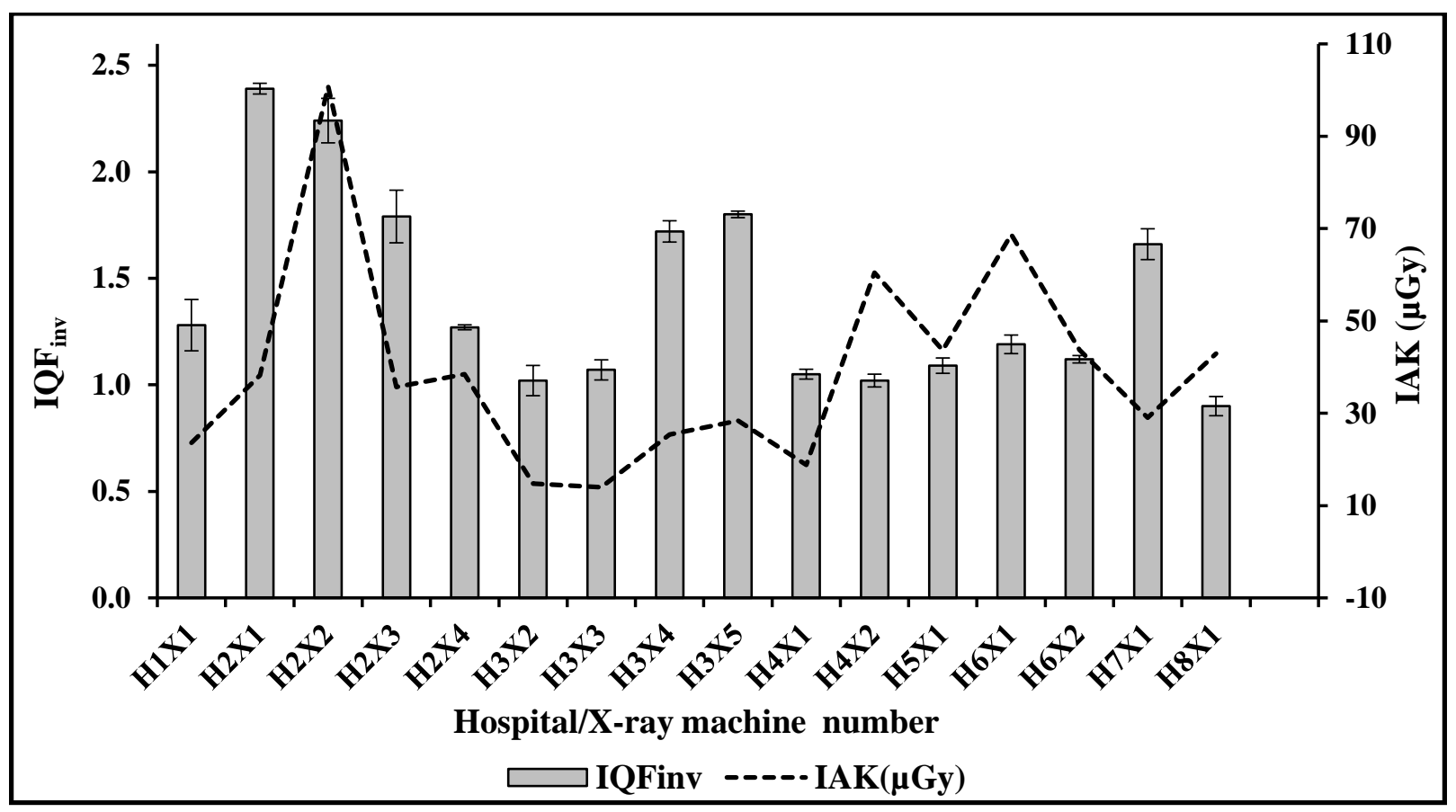


Fig. 5. A comparison of $I Q F_{\text {inv }}$ against the IAK for the ten years age group, between /within hospitals. $\mathrm{H}=$ hospital; $\mathrm{X}=\mathrm{X}$-ray unit. The bars represent the $\mathrm{IQF}_{\text {inv }}$ values with the accompanying error bars representing the SD of three replicated images. The dotted line represents the IAK values. 
Table 1. A summary of X-ray machine types and examination techniques used for paediatric CXR (between/within hospitals).

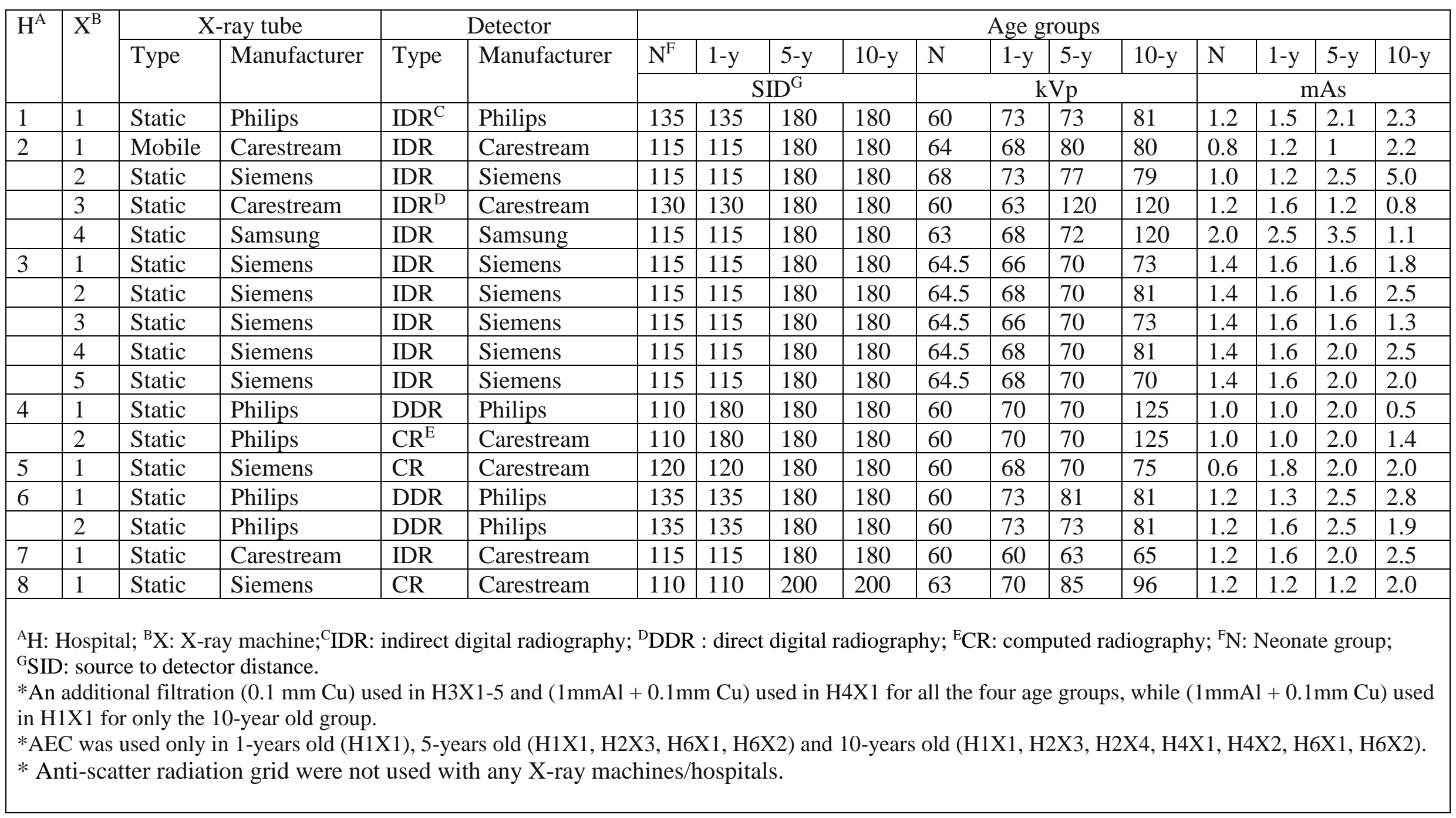


Table 2. Details of the variation in IAK and $I_{Q} F_{\text {inv }}$ for the four peadiatric age groups, both between and within the hospitals.

\begin{tabular}{|c|c|c|c|c|c|c|c|}
\hline \multirow{3}{*}{$\begin{array}{l}\text { Age } \\
\text { groups }\end{array}$} & \multicolumn{4}{|c|}{ Between the hospitals } & \multicolumn{3}{|c|}{ Within the hospitals } \\
\hline & Minimum & Maximum & $\begin{array}{l}\text { Percentage } \\
\text { difference }\end{array}$ & $\begin{array}{l}\text { Mean/ } \\
\text { Median }\end{array}$ & Minimum & Maximum & $\begin{array}{l}\text { Percentage } \\
\text { difference }\end{array}$ \\
\hline & \multicolumn{7}{|c|}{ IAK } \\
\hline Neonate & 8.56 & 52.62 & $144.0 \%$ & $24.93 *$ & 21.79 & 52.62 & $82.9 \%$ \\
\hline 1-year & 5.44 & 82.82 & $175.3 \%$ & $36.84 *$ & 36.78 & 82.82 & $77.0 \%$ \\
\hline 5-year & 10.97 & 59.22 & $137.5 \%$ & $21.43 * *$ & 11.75 & 52.94 & $127.3 \%$ \\
\hline \multirow[t]{2}{*}{ 10-year } & 13.97 & 100.77 & $151.3 \%$ & $35.72 * *$ & 35.72 & 100.77 & $95.3 \%$ \\
\hline & \multicolumn{7}{|c|}{ IQFinv } \\
\hline Neonate & 1.40 & 4.44 & $104.1 \%$ & $2.58 *$ & 2.45 & 4.44 & $57.8 \%$ \\
\hline 1-year & 0.96 & 4.73 & $132.5 \%$ & $2.39 *$ & 2.33 & 4.73 & $68.0 \%$ \\
\hline 5-year & 0.87 & 1.81 & $70.1 \%$ & $1.35^{*}$ & 0.98 & 1.46 & $39.3 \%$ \\
\hline 10-year & 0.90 & 2.39 & $90.6 \%$ & $1.23 * *$ & 1.27 & 2.39 & $61.2 \%$ \\
\hline
\end{tabular}

